Accepted version of paper:

\title{
Packaging Challenges and Reliability Performance of Compound Semiconductor Focal Plane Arrays
}

Authors: Stoyan Stoyanov, Chris Bailey, Rhys Waite, Christopher Hicks and Terry Golding

Conference: 2019 22nd European Microelectronics and Packaging Conference \& Exhibition (EMPC), Pisa, Italy, 16-19 Sept. 2019

Date Added to IEEE Xplore: 09 January 2020

GALA Repository Upload Date: 22/01/2020

This is IEEE-copyrighted Article. Published version available from IEEE Xplore:

DOI: $10.23919 /$ EMPC44848.2019.8951842 


\title{
Packaging Challenges and Reliability Performance of Compound Semiconductor Focal Plane Arrays
}

\author{
Stoyan Stoyanov ${ }^{1}$, Chris Bailey ${ }^{1}$, Rhys Waite ${ }^{2}$, Christopher Hicks ${ }^{2}$ and Terry Golding ${ }^{3}$ \\ ${ }^{1}$ Computational Mechanics and Reliability Group, University of Greenwich, London, UK \\ ${ }^{2}$ Microchip Technology Inc., Caldicot, UK \\ ${ }^{3}$ Amethyst Research Inc., Ardmore, OK, USA \\ Phone: +44 (0)20 83318520 E-mail: s.stoyanov@gre.ac.uk
}

\begin{abstract}
The development of new high-performance Focal Plane Arrays (FPAs) for imaging systems is driven by advances in photodetector material growth and processing, readout integrated circuits and IR detector chip hybridisation/packaging. The hybridisation of the IR detector chip and the readout integrated circuit (ROIC) through flip-chip bonding is a key packaging challenge for pixel arrays with very small indium bumps and 10-30 $\mu m$ pitch sizes. This paper details the development and use of finite element models that can be used to assess and optimise the compression bonding process, and can enable insights into the impact of chip misalignment on the resulting flip-chip quality and the bonding equipment placement accuracy requirements for a given FPA specification. In addition, the fatigue performance of the indium interconnects of different fine pitch FPAs is evaluated and compared. The modelling results point that high quality interconnects and robust, defects-free assembly require micrometre placement accuracy. It is also possible that indium joints of higher resolution, larger size FPAs accumulate less damage under cryogenic temperature cycling compared to less dense, smaller in size, focal plane arrays.
\end{abstract}

Key words: Finite element modelling, focal plane array, compression bonding, flip-chip assembly, reliability, cryogenic temperature, compound semiconductors.

\section{Introduction}

Focal plane arrays (FPAs) are image sensing devices featuring typically a two-dimensional rectangular array of light-sensing pixels. They have been traditionally developed for military applications such as target acquisition, night vision, and weapon and missile seekers. In the past few decades, there has been substantial research and development in the area of IR detectors, particularly in relation to new detector materials and in the design and fabrication of very fine pitch, high resolution pixel array structures [1-3]. At present, FPAs are increasingly designed, fabricated and used in a wide range of non-military applications, from medical diagnostics and industrial process control to security and surveillance to astronomy and civil space applications [4-7]. There is a strong and emerging interest to expand the use of FPAs into the commercial market.

The operating principle of FPAs involves the pixels detection of incoming infrared radiation and conversion of photons into respective electrical signals in order to form an image. The focal plane array comprises of two parts: (1) the IR detector array and (2) the readout integrated circuit. The detector pixel array is the infrared-sensing element of the focal plane array and the readout integrated circuit is the signal processing component.
The IR detector and FPA technology developments are driven by demands for improved sensitivity and resolution of the imaging systems, needs for high-yield low-cost fabrication capabilities, and continuing advances in the sensing material compositions and their processing $[8,9]$. One of the main hurdles that is yet to be overcome is the establishment of supply chains for FPAs production and use within a wider commercial market.

The FPA packaging is also known as hybridisation. It is among the key challenges for the industry. This is the process of the physical assembly through flip-chip bonding and electrical integration of the IR detector chip and the ROIC. Indium solder is the industry standard for FPA interconnection material. The hybridisation concept/process directly impacts yield, reliability and reproducibility, and dictates the ability and level of success to which high resolution and small pitch size pixel arrays can be handled. Higher stand-off height and higher uniformity of the indium bumps have been pursued in FPA fabrication, and as a result manufacturing techniques for indium bumping of IR detectors and ROIC chips have been extensively researched and developed [10].

Advanced FPAs feature high density pixel/interconnect arrays utilising very small size indium bumps and chip contact pads which make the 
assembly of the detector chip onto the readout integrated and hence requires advanced, high precision placement equipment. The reason for this is that achieving high quality interconnection between the detector pixel matrix and the readout chip is a critical requirement for the proper functional performance of the imaging device.

In this paper, a computational approach utilising finite element models is developed and used to provide critical assessments of the technical challenges and requirements associated with the alignment accuracy and the compression bonding flip-chip process for the packaging of highresolution, large pixel array imaging IR detector chips. The bonding process models are used for quantitative evaluation of the effects of inaccurate placement of the detector chip onto the ROIC, and enabled insights into the resulting indium joint characteristics such as shape and stand-off height and the risks for poor interconnection quality.

In addition, the thermo-mechanical fatigue damage of the indium solder interconnects in assembled FPAs under thermal cycling at cryogenic temperatures (300K to $77 \mathrm{~K}$ ) is also assessed. Most IR FPA are integrated with a cryocooler to provide operating temperature of the device below $150 \mathrm{~K}$. This is required in order to reduce the thermallyinduced noise and the noise-to-signal ratio and thus improve dramatically the FPA spatial resolution and sensitivity characterises. As liquid nitrogen is the most common cooling solution, operating temperature of $77 \mathrm{~K}$ defines the thermal load considered in this investigation. The modelling study aimed to generate new insights into the reliability performance trends of FPAs when respective resolution/pixel matrix size increase and I/O pitch sizes decrease.

\section{FPA Specifications: Geometry and Materials}

\section{Geometric Data}

IR imaging sensors with pixel arrays of $320 \times 256,640 \times 512$ and $1280 \times 1024$ are investigated. The respective FPAs feature pitch size in the range $12 \mu \mathrm{m}$ to $30 \mu \mathrm{m}$, indium interconnect volumes from $0.896 \mathrm{E}-6 \mathrm{~mm}^{3}$ to $1.4 \mathrm{E}-6 \mathrm{~mm}^{3}$, and contact pad sizes in the range of 8-10 $\mu \mathrm{m}$. An outline of a typical IR detector chip/ pixel array structure, with details for the mesa pixel and pad layout are presented in Fig. 1. In addition, this figure shows also a schematic of the pre-bonded flip-chip stack of the detector and the ROIC chips. Larger in size non-pixel peripheral joints (supported by respective pad layouts) complete the FPA assembly interconnection requirements.

The key specifications of the three investigated high resolution FPAs are provided with Table 1. For example, the 1280x1024 IR detector chip has pitch size $12 \mu \mathrm{m}$ and mesa pixel size $9 \times 9$ $\mu \mathrm{m}$, with a $3 \mu \mathrm{m}$ gap between two adjacent pixels.
The contact pads and the mesa pixels have submicron thickness. The contact pads on the ROIC and the detector chips have square shape with dimensions as reported in Table 1 .

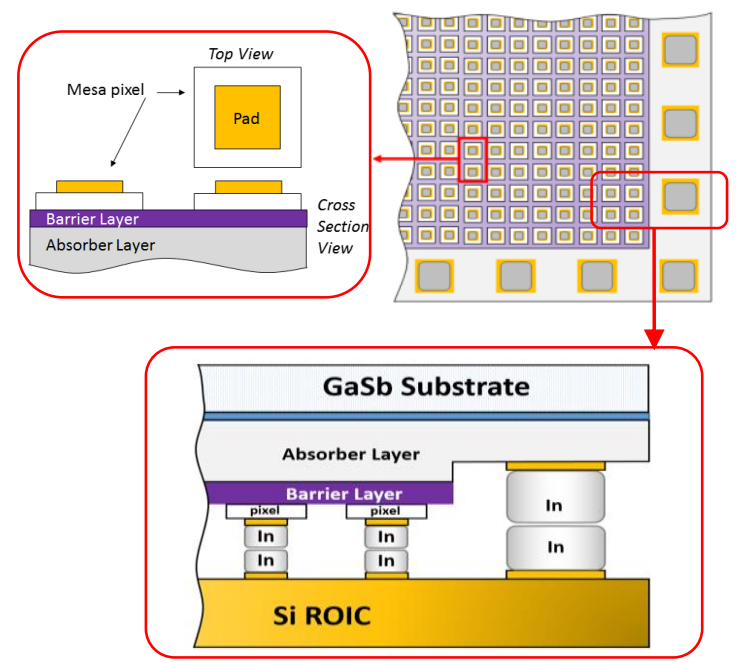

Figure 1: IR detector pixel array outline and schematic of the pre-bonded flip-chip assembly (IR detector and ROIC chips).

Table 1: FPA specifications.

\begin{tabular}{lccc}
\hline \hline FPA Assembly & FPA \#1 & FPA \#2 & FPA \#3 \\
\hline \hline Pixel Array & $320 \times 256$ & $640 \times 512$ & $1280 \times 1024$ \\
\hline No. of pixels & 81,920 & 327,680 & $1,310,720$ \\
\hline Pixel Pitch $(\mu \mathrm{m})$ & 30 & 20 & 12 \\
\hline Pixel size $(\mu \mathrm{m})$ & $25 \times 25$ & $17 \times 17$ & $9 \times 9$ \\
\hline Contact pads $(\mu \mathrm{m})$ & $10 \times 10$ & $10 \times 10$ & $8 \times 8$ \\
\hline ROIC Chip & FLIR & FLIR & FLIR \\
Reference & ISC9809 & ISC0402 & ISC1308 \\
\hline \hline
\end{tabular}

As detailed in Table 1, commercial off-theshelf ROIC chips from the infrared and imaging systems company FLIR have matching specifications for the three detector pixel array format resolutions. For example, the readout integrated circuit for the assembly of the $1280 \times 1024$ IR detector chip is the FLIR ISC1308 ROIC [11]. This ROIC has a $1280 \times 1024$ contact pads matching layout at $12 \mu \mathrm{m}$ pitch size.

The IR detector chips are Group III-V compound semiconductor materials. The gallium antimonide $(\mathrm{GaSb})$ substrate of the detector chip is a pre-assembly feature only and commonly designed to have thickness comparable with the thickness of the ROIC. Once the FPA hybrid stack is formed and the IR chip is bonded onto the ROIC, the GaSb substrate is removed. Thus, the photo-sensing chip in a final FPA is only few microns tick, comprising the absorber layer, barrier layer and the mesa pixels only as detailed in Fig. 1. A solder joint is assumed to be obtained by two pre-bumped indium deposits on the IR detector chip and the ROIC pads respectively. For the discussed assemblies in this work, the indium deposits are equally split volume- 
wise between the opposing pads of the flip-chip structure (see Fig 1, bottom).

\section{Material Data}

The numerical (finite element) modes for the analysis of the FPA assembly bonding processes and the thermo-mechanical FPA reliability performance under cryogenic temperature cycling loads require also material data in addition to the geometric and topology data of the flip-chip hybridised chips. In the models, FPA materials, excluding the indium solder, are considered as having elastic behaviour. Temperature dependent data, where available, is included in the respective models. The required material properties for the ROIC chip (Si), IR detector chip substrate $(\mathrm{GaSb})$, contact pads $(\mathrm{Au})$ and interconnect material (In) include Young's modulus, Poisson's ratio and coefficient of thermal expansion (CTE). These are summarized in Table 2. The utilized linear coefficient of thermal expansion data for silicon and indium is sourced from reference [12]. The absorber layer, pixel and barrier layer materials, as illustrated in Fig. 1, are all Group III-V compound semiconductor materials with values for Young's modulus, Poisson's ratio and CTE in the range of $62 \mathrm{MPa}, \quad 0.32$ and 5.5-5.9 $\mathrm{ppm} / \mathrm{K}$ respectively.

The material behaviour of the indium solder in particular is very important for the utilized simulation models. The indium visco-plastic behaviour is modelled using the Anand inelastic strain rate model [13].

Table 2: Material data used in models.

\begin{tabular}{|c|c|c|c|}
\hline Material & $\begin{array}{l}\text { Young's } \\
\text { Modulus } \\
\text { (GPa) }\end{array}$ & $\begin{array}{c}\text { Poisson's } \\
\text { Ratio }\end{array}$ & $\begin{array}{c}\mathbf{C T E} \\
\left(\mathbf{p p m} \cdot \mathbf{K}^{-1}\right)\end{array}$ \\
\hline Indium (In) & $\begin{array}{l}20.54 @ 77 \mathrm{~K} \\
16.24 @ 187 \mathrm{~K} \\
12.70 @ 300 \mathrm{~K}\end{array}$ & $\begin{array}{l}0.433 @ 77 \mathrm{~K} \\
0.441 @ 187 \mathrm{~K} \\
0.449 @ 300 \mathrm{~K}\end{array}$ & $\operatorname{Ref}[12]$ \\
\hline Silicon $(\mathrm{Si})$ & 168.0 & 0.28 & \\
\hline Gold $(\mathrm{Au})$ & 77.2 & 0.42 & 14.4 \\
\hline $\begin{array}{l}\text { Gallium } \\
\text { antimonide } \\
(\mathrm{GaSb})\end{array}$ & 76.0 & 0.30 & $\begin{array}{l}1.4 @ 77 \mathrm{~K} \\
4.5 @ 140 \mathrm{~K} \\
6.0 @ 200 \mathrm{~K} \\
6.5 @ 300 \mathrm{~K} \\
\end{array}$ \\
\hline
\end{tabular}

\section{Compression Bonding Modelling of FPAs}

Compression bonding is the most common hybridisation technique for of the IR detectors because of the indium's ductile and malleable material behaviour. The ability to model the compression bonding of FPAs can be very beneficial. It can help the assessment of the effects of chip misalignment on the resulting feasibility and quality of indium bump formation, and can be also used to provide model-based capability for process optimisation. These benefits are demonstrated in this section of the paper.

\section{Modelling Approach}

The modelling approach is developed to enable the assessment of the impact which applied compression loads on the IR detector chip have on the indium joint formation process. Finite element method and ANSYS simulation software [14] are employed to develop two-dimensional finite element model of the FPA assembly at local level, representing one pixel unit spatial domain and the formation of a single joint within the full assembly array.

Figure 2 details the finite element model of the FPA for simulation of the compression bonding condition and the application of the respective model boundary conditions (BC): (1) the applied pressure (i.e. bonding force) and (2) the structural constraints (displacement degrees-of-freedom, DOF) along with the contact boundary for the indium-to-indium joint formation. The contact boundary is defined over the regions where the expected (i.e. IR detector chip indium bump to ROIC indium bump contact boundary) or potential (indium to vicinity of the pad area) contact may take place during bonding. ANSYS contact pair (element types TARGE169 and CONTA172) modelling capability is exploited, with "no separation" condition for the behaviour of the contact surface and friction coefficient 0.2.

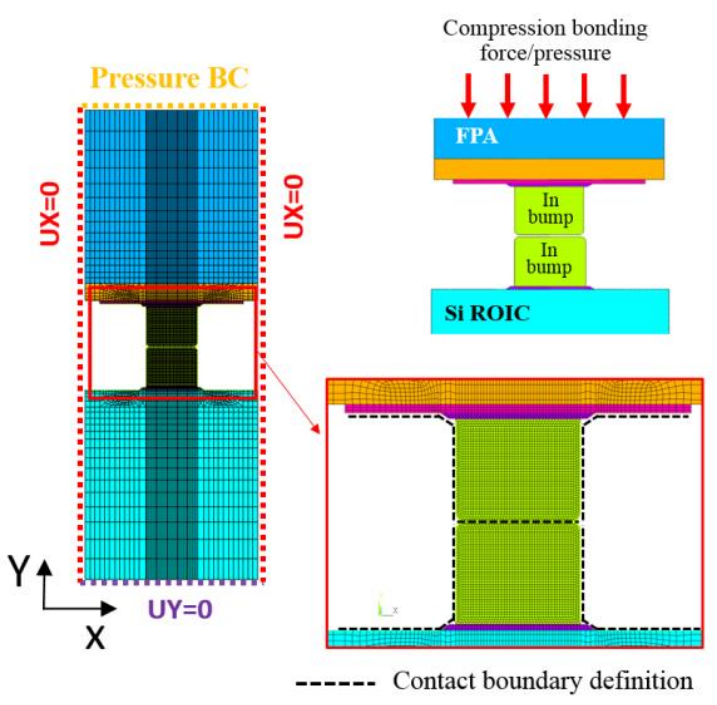

Figure 2: Finite element model for compression bonding simulation of focal plane arrays.

Non-linear transient simulations are undertaken under the conditions for large deformation. The formation of the single indium joint is a result of a diffusion process (not simulated here) at the established contact interface of the two indium deposits and under sustained bonding force that is applied. The developed model assumes that there is no lateral movement of the flipped chip during bonding. With this study, the modelling results are obtained under the assumption for bonding process undertaken at room temperature. 
The applied compression bonding load profile consists of the phases. The pressure is first ramped linearly from 0 to the load profile peak value $\left(\mathrm{P}_{\max }\right)$, and then, in the second phase, the pressure is maintained constant at that level for the remaining duration of the load application. The reported results are obtained with a load profile where the ratio of pressure ramp up time to constant pressure time is 1:5. The applied pressure (or force) peak value and the ramp up/dwell durations of the load application are process parameters that need to be uniquely optimised for a given FPA so that the shape and the bonding quality of the resulting indium joint at the end of the applied load profile meet specification requirements.

\section{Modelling Results}

An accurate placement of the IR detector chip onto the ROIC chip so that respective contact pads on both sides for the array of indium joints match is the key requirement to enable the assembly by means of applying a compression force. With the developed process model, it is possible to evaluate how different placement accuracy may impact the formation of the indium joints. The accuracy of placement of the IR detector chip can be defined with the value of $\Delta p$ (see Fig. 3, top) which measures the offset between two corresponding pads (on the ROIC and IR detector sides). Here, two different levels of misalignment are assessed; referred to as Cases A and B (see Fig. 3, bottom):

- Case A: Exact positioning $(\Delta \mathrm{p}=0 \mu \mathrm{m})$

- Case B: Moderate misalignment $(\Delta \mathrm{p}=3 \mu \mathrm{m})$.

Below, the modelling predictions are demonstrated only in the case of bonding the $320 \times 256$ FPA. Analysis of misalignment effects for any other FPA, with different pixel resolution and geometric specification, and using the same process model can be performed in a very similar way.

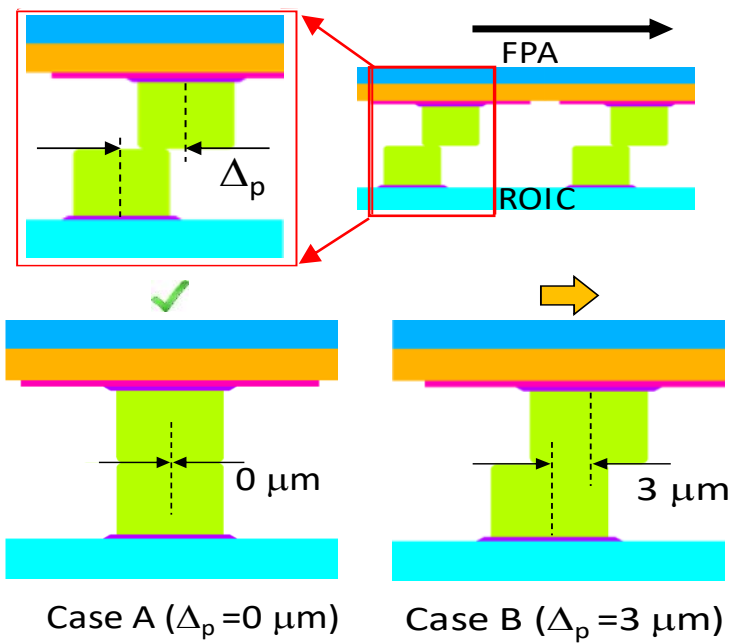

Figure 3: Simulated misalignment cases for the compression bonding of the $320 \times 256$ FPA.
Figure 4 details the shape deformation of the indium joint assuming the placement accuracy scenarios (Cases A and B). The last row details the deformed shape of the final joint achieved at the end of the applied bonding profile. It should be noted that due to the inelastic (visco-plastic) behaviour of indium, the deformation of the formed joint takes place throughout the entire application of the bonding pressure/force, including during the phase of the profile where the applied pressure is kept constant. The final shape of the formed joint is achieved at the end of the compression profile when the pressure from the bonding force is removed.

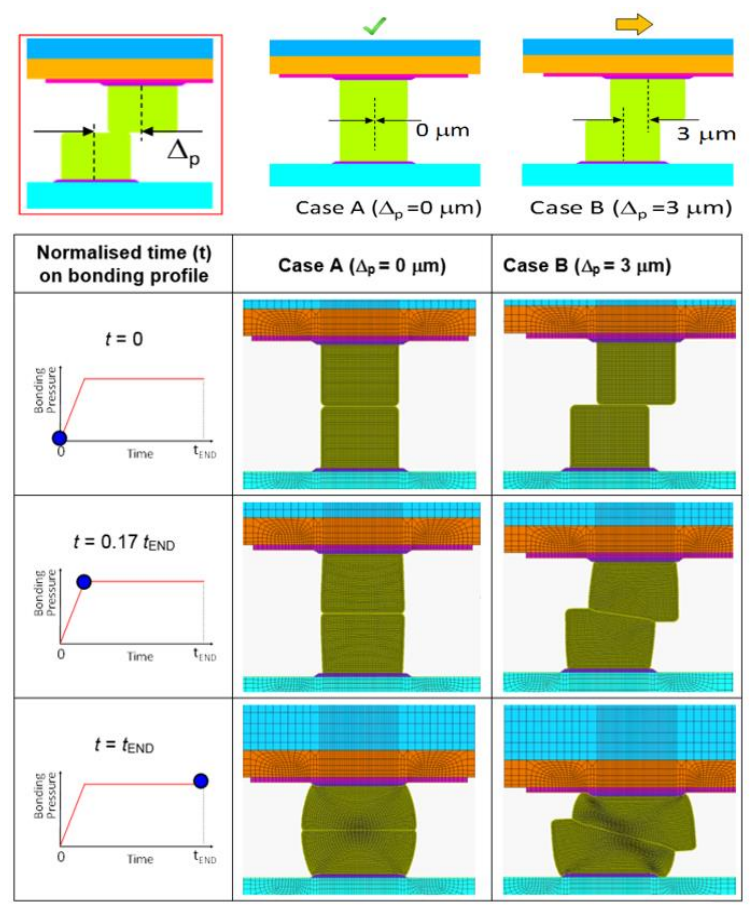

Figure 4: Simulations results from compression bonding analysis of the effect of placement accuracy on indium joint formation for $320 \times 256$ FPA.

The main conclusion is that misalignment of even few microns may be difficult to accept and tolerate at such small dimensions of the formed interconnects. In this instance, a misalignment of 3 $\mu \mathrm{m}$ may still result in the formation of a joint but, as evident from the modelling results, the indium joint shape is compromised quality-wise and the interconnect comes with a very uncertain (variable) and significantly lower stand-off height. Presence of such attributes mean that the FPA performance and expected reliability will be compromised. Although for this specific FPA assembly there is no risk of bridging between adjacent joints, misalignment is also problematic in the context of implementing a robust control of the bonding force magnitude needed to avoid excessive indium bump collapse and avoid the risk of joint formation outside the area of the contact pad.

It is clear that the formation of joints is highly sensitive not just to the placement accuracy of the IR 
detector chip onto the ROIC chip but also to the bonding profile parameters: (1) the maximum pressure/binding force, and (2) the load ramp and dwell time durations. Because the process model is capable of assessing the magnitude of the joint deformation during bonding, it can be used to support the bonding process optimization for given equipment and alignment accuracy.

An example of such model use can be given by assessing the compression load magnitude changes required in the case of the three FPAs discussed in this work. This is because the FPAs have different pixel array density and additional geometric variations. Assuming perfectly aligned flip-chips for the bonding of the $320 \times 256,640 \times 512$ and 1280x1024 FPAs, respective process models were employed and used to identify the required bonding pressure condition that can achieve a predefined target stand-off height value of $9.5 \mu \mathrm{m}$ for the indium joints. For the analysed compression bonding profile with duration indicated by the normalised time value $t_{\mathrm{END}}$ and ratio of load ramp up time to dwell time at peak pressure 1:5, the optimal maximum pressure value $\left(\mathrm{P}_{\max }\right)$ for the compression bonding has scaled across the three focal plane arrays as follows:

$$
\begin{aligned}
& \text { - 320x256 FPA: } \quad \mathrm{P}_{\max }=\mathrm{P}_{\text {nom }} \text {; } \\
& \text { - 640x512 FPA: } \mathrm{P}_{\max }=1.5 \mathrm{P}_{\text {nom; }} \text {; } \\
& \text { - 1280x1024 FPA: } \mathrm{P}_{\max }=2.0 \mathrm{P}_{\text {nom }} \text {. }
\end{aligned}
$$

These results show that compression bonding set up is not trivial and changes substantially as IR detector arrays change specification. The process of identifying the optimal process parameters of the compression bonding profile can be greatly aided by adopting the discussed process models.

\section{Reliability Modelling of FPAs}

The thermo-mechanical modelling of the thermal fatigue damage in indium joints of FPAs under cryogenic temperature cycling load conditions can aid the qualitative analysis for the expected reliability performance for different high resolution focal plane arrays. Here, such demonstration is detailed for the three FPA's being investigated. As no failure data for indium joints of FPA structures and respectively no life-time model are available, at present the reported model predicted damage induced in the indium joints of the FPA is not correlated to corresponding cycles to failure.

\section{Thermo-mechanical modelling}

A thermo-mechanical fatigue model for each of the three high resolution FPAs is constructed as a full three-dimensional (3D) slice model. A model captures explicitly all assembly details in a crosssectional slice along the diagonal line of the FPA. Note that the GaSb substrate is only a pre-assembly feature of the detector array and therefore once the assembly (hybridization) is completed, the substrate is removed and is not part of the final FPA. Due to existing symmetry along the diagonal line of the FPA, the slice model includes the domain from the central (neutral) point of the assembly to the corner of the ROIC chip, through the whole assembly thickness.

The slice modelling approach is superior to two-dimensional models and is an appropriate, accurate enough, modelling strategy when full 3D model is not practical (as in this case due to extremely large pixel arrays). Figure 5 illustrates the slice model developed for the 320x256 FPA, with similar models also developed for the FPAs with resolutions $640 \times 512$ and $1280 \times 1024$ pixels. The figure provides also a detailed view on the finite element mesh and the geometric representation at the level of a single solder joint in the assembly. These models assume no misalignment of the flipped IR detector chip and thus perfectly formed shape-wise indium solder joints.

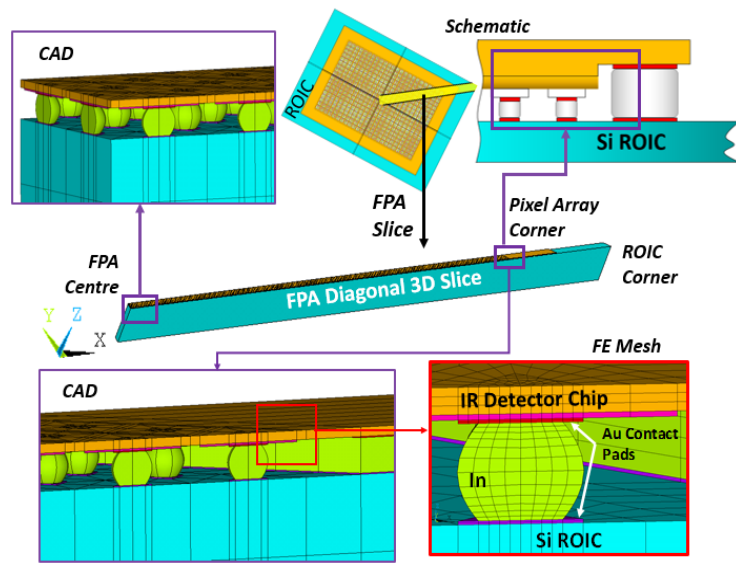

Figure 5: 3D slice model illustration developed for the fatigue analysis of indium joints in 320x256 FPA.

Non-linear transient thermo-mechanical analysis that simulates the responses of the FPAs under applied (imposed isothermal) temperature cycling load is undertaken using ANSYS simulation software. The domain is meshed with element type SOLID185. The range of the temperature cycle is $293 \mathrm{~K}$ to $77 \mathrm{~K}$ over 1 hour, with 15 minutes ramp/dwell times. Mesh size of the developed 3D slice models for the three FPAs is in the range 330,000 to 575,000 mesh elements. Standard for a 3D diagonal slice model structural boundary conditions are applied: (1) for the displacement degree-of-freedom (DOF) in perpendicular direction to the slice we have symmetry on the "front" face of the slice and coupled degree-of-freedom (out-ofslice plane) for the nodes on the "back" side of the slice, (2) all DOF fixed at a point along the central line of the assembly, and (3) symmetry at the face of the slice defining half-diagonal symmetry.

The low cycle thermal fatigue, being driven by the accumulation of permanent deformation (i.e. 
inelastic strain) experienced by the solder joints, can be evaluated with the simulation models by predicting the plastic work per unit volume in the solder joints. The fatigue damage indicator is therefore defined as the accumulated visco-plastic strain energy density accumulated in the indium joints over one temperature cycle. The damage indicator, denoted as $\Delta W_{\text {ave }}$, is calculated as an average of the accumulated visco-plastic strain energy density (over one temperature cycle) in an interfacial layer of the indium joint with the contact pad where the highest damage is predicted, and hence where the crack is expected to initiate and propagate. This calculation is undertaken on the most stressed joint, expected and confirmed to be the corner one in the array for the studied FPAs, with layer thickness for the averaging result $0.5 \mu \mathrm{m}$. Such averaging approach is best practice as point results can be influenced by finite element mesh sizes and stress singularity effects.

\section{Modelling results for thermal fatigue damage in indium joints}

The thermo-mechanical simulation results show that with all three FPA structures the most critical (most damaged) joint in the array is the one located at the corner. The maximum concentration of the accumulated inelastic energy density is found at the interface of the joint with the contact pad on the ROIC side of the package, and at the pad region facing towards the centre of the assembly (inwards). The simulation results for the predicted thermal fatigue damage are illustrated in Fig. 6.

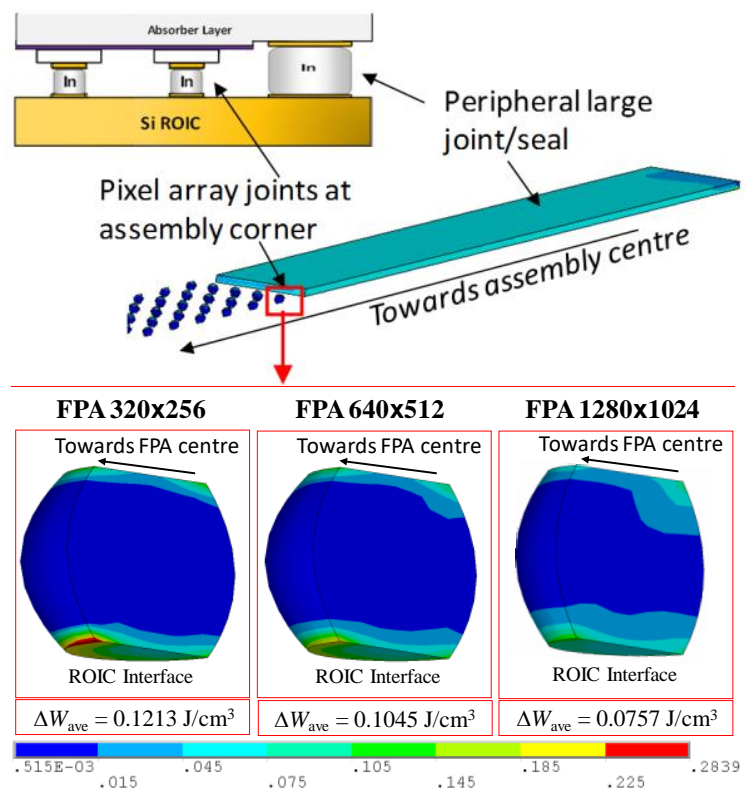

Figure 6: Simulation results for the thermal fatigue damage of indium solder joints in the form of plastic work $\left(\mathrm{J} / \mathrm{cm}^{3}\right)$ accumulated over one temperature cycle. The results detail the state of the most critical corner solder joint of the studied high-resolution FPA assemblies.
Based on the predictions obtained with the finite element models, the damage parameter $\left(\Delta W_{\text {ave }}\right)$ for the critical indium joint is found to be:

- $14 \%$ lower with the 640x512 FPA compared to the $320 x 256$ FPA assembly;

- $38 \%$ lower with the 1280x1024 FPA compared to the $320 \times 256$ FPA assembly.

Figure 7 provides a graph (point result where the peak plastic work is found) that details how the damage accumulates over the temperature cycle. The damage is predominantly induced as the temperature load is ramped, up or down, while much smaller accumulation of damage takes place during the dwell periods of the cycle.

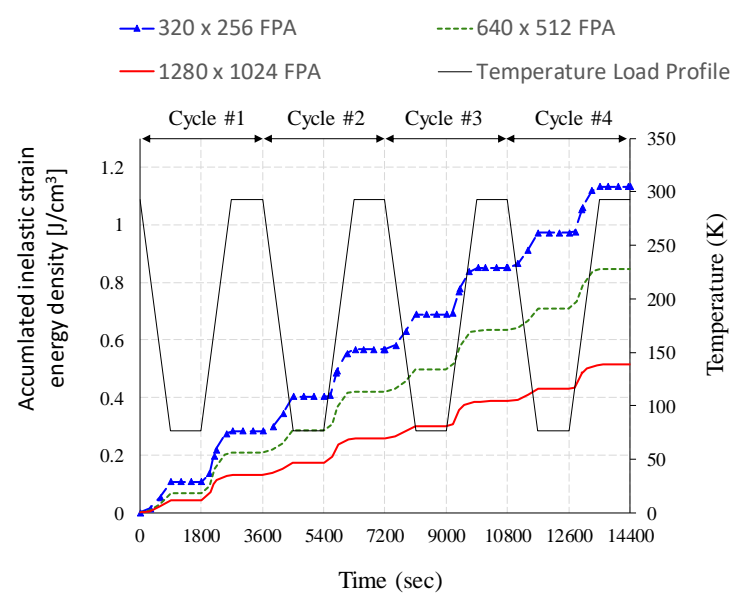

Figure 7: Accumulation of damage in indium joints during cryogenic temperature cycling maximum inelastic energy density point result associated with the critical corner indium joint of the FPA assemblies.

These thermo-mechanical results for indium solder damage show that as the pixel array increases its resolution and density, and despite the fact that the size of the assembly gets larger (and thus the corner joint gets further away from the assembly's neutral point), the damage in the indium joints in the array, including the one at the corner, is getting smaller. The above finding is important and is not trivial. It can be only confirmed either through the presented modelling approach or by undertaking actual experimental tests of thermal cycling on the three FPA assemblies.

The reason for the finding that more complex, high density pixel array assemblies may potentially be more reliable in the context of the thermal fatigue reliability of the indium solder interconnects is this: the shear strain impact from the in-plane linear CTE mismatch between the ROIC and the detector chip is accommodated better as the array size increases and this causes the shear strain per joint to get smaller. This can be seen as an improved ROIC - IR detector chip compliance that can be potentially featured by larger pixel arrays. 
It should be noted that the $1280 \times 1024$ FPA joints have smaller interfacial area with the contact pad and hence in real terms a crack will need to propagate over smaller distance to cause the failure. While this is an implication for the actual time-tofailure, the damage prediction itself will still imply having the slowest rate of crack initiation and propagation with the high risk FPA.

The large peripheral indium interconnect is found to play, in addition to the electrical signalling functional role, an important structural role. Simulation results not detailed in this paper reveal that the larger peripheral joints reduce the value of the predicted thermal fatigue damage, $\Delta W_{\text {ave }}$, in the corner solder join of the array by as much as 75$80 \%$. The damage in the large peripheral joint itself should not be of a concern as the damage is at lower or similar level as for the corner array joint but the length of the crack to fail the joint is greater by factor of $50(8-10 \mu \mathrm{m}$ for the array joint vs $>500 \mu \mathrm{m}$ for the peripheral joint).

\section{Conclusions}

Finite element models for the compression bonding process of flip-chip hybridisation of high resolution IR detector chips with readout integrated circuits and for the thermo-mechanical reliability performance of the resulting FPA fine-pitch indium solder joints have been developed and demonstrated. The models enable critical capabilities for the optimisation of compression bonding process parameters and assessing the flip-chip alignment requirements as well as supporting FPA design-forreliability activities.

The main findings from the undertaken studies in relation to the IR detector and the ROIC chip hybridisation are as follows:

- Misalignment of the IR detector chip onto the ROIC in the flip-chip bonding process has major impact on the quality of the resulting joints in terms of shape, out-of-pad contact and most importantly the expected stand-off height. This can affect the long-term performance and reliability of the focal plane array.

- Uncertainty in placement accuracy makes the optimal compression bonding parameter setup problematic as different levels of misalignment result in different characteristics of the indium joints under same compression bonding conditions. This makes practically impossible to adopt a robust assembly condition that can assure high quality of the resulting FPAs.

- Assembly equipment capable of providing accuracy of chip placement $<3 \mu \mathrm{m}$ and better is a key requirement.

In terms of the fatigue reliability of the indium interconnects of FPAs under temperature cryogenic cycling loads, the modelling study enabled to conclude:
- The most damaged (corner) joints in higher resolution FPAs may accumulate less damage compared to less dense focal plane arrays. This can happen despite the fact that the higher resolution FPAs may be larger in size and thus have the corner joints at larger distance from the neutral (FPA centre) point of the assembly.

- Although the above result was observed in this work, it needs to be model-confirmed in each instance of a given FPA structure. This is because the shear force distribution depends on both the total number of interconnects and the actual physical land size of the matrix array (respectively the pitch size).

\section{Acknowledgements}

This work has been funded by Innovate-UK through the "Technically high element alignment (THEIA)" project, project number 103439.

\section{References}

[1] P. Martyniuk et al., "New concepts in infrared photodetector designs", Applied Physics Reviews, Vol. 1, Issue 4, pp. 1-35, 2014.

[2] R. Zhao, W. Ma, S. Wang, X. Yu, Y. Feng and Y. Zhao, "Design and fabrication of a sandwich framed focal plane array for uncooled infrared imaging", Proc. 18th International Conference on Solid-State Sensors, Actuators and Microsystems, Anchorage, AK, USA, June 21-25, pp. 1318-1321, 2015.

[3] Y. Ou et al., "Design, fabrication, and characterization of a $240 \times 240$ MEMS uncooled infrared focal plane array with $42-\mu \mathrm{m}$ pitch pixels", Journal of Microelectromechanical Systems, Vol. 22 , No. 2, pp. 452-461, 2013.

[4] C. Downs and T.E. Vandervelde, "Progress in infrared photodetectors since 2000", Sensors, Vol. 13, pp. 5054-5098, 2013.

[5] A. Rogalski, "Infrared detectors for the future", Acta Physica Polonica, A, Vol.116, No. 3, pp. 389-406, 2009.

[6] Antoni Rogalski, "Infrared Detectors", Taylor \& Francis, Boca Raton, 2nd edition, 2011.

[7] T. Sprafke and J.W. Beletic, "High-performance infrared Focal Plane Arrays for space applications", Optics and Photonics News, Vol. 19, Issue 6, pp. 22-27, 2008.

[8] A. Karim and J.Y. Andersson, "Infrared detectors: Advances, challenges and new technologies", IOP Conference Series: Materials Science and Engineering, Vol. 51, 01200, pp. 1-8, 2013.

[9] A. Rogalski, "Next decade in infrared detectors", Proc. SPIE Vol. 10433, ElectroOptical and Infrared Systems: Technology and Applications XIV, 104330L, 2017.

[10] J. Jiang et al., "Fabrication of indium bumps for hybrid infrared focal plane array applications", 
Infrared Physics \& Technology, Vol. 45, Issue 2, pp. 143-151, 2004.

[11]FLIR, https://www.flir.co.uk/products/isc9809/

[12]X. Cheng, C. Liu and V.V. Silberschmidt, "Numerical analysis of thermo-mechanical behavior of indium micro joint at cryogenic temperatures", Computational Materials Science, Vol. 52, pp. 274-281, 2012.

[13] R.W. Chang and F.P. McCluskey, "Constitutive relations of indium in extreme temperature electronic packaging based on Anand model", Journal of Electronic Materials, Vol. 38, No. 9, pp. 1855-1859, 2009.

[14] ANSYS, www.ansys.com 